\title{
Microcirculatory perfusion disturbances in septic shock: results from the ProCESS trial

Michael J. Massey ${ }^{1}$, Peter C. Hou², Michael Filbin ${ }^{3}$, Henry Wang ${ }^{4}$, Long Ngo ${ }^{5}$, David T. Huang ${ }^{6}$, William C. Aird ${ }^{7}$, Victor Novack ${ }^{8}$, Stephen Trzeciak ${ }^{9}$, Donald M. Yealy ${ }^{10}$, John A. Kellum ${ }^{6}$, Derek C. Angus ${ }^{6}$, Nathan I. Shapiro ${ }^{*^{*}}$ (D) and for the ProCESS investigators ${ }^{7}$

\begin{abstract}
Background: We sought to determine the effects of alternative resuscitation strategies on microcirculatory perfusion and examine any association between microcirculatory perfusion and mortality in sepsis.

Methods: This was a prospective, formally designed substudy of participants in the Protocolized Care in Early Septic Shock (ProCESS) trial. We recruited from six sites with the equipment and training to perform these study procedures. All subjects were adults with septic shock, and each was assigned to alternative resuscitation strategies. The two main analyses assessed (1) the impact of resuscitation strategies on microcirculatory perfusion parameters and (2) the association of microcirculatory perfusion with 60-day in-hospital mortality. We measured sublingual microcirculatory perfusion using sidestream dark field in vivo video microscopy at the completion of the 6-h ProCESS resuscitation protocol and then again at 24 and $72 \mathrm{~h}$.
\end{abstract}

Results: We enrolled 207 subjects (demographics were similar to the overall ProCESS cohort) and observed 40 (19. 3\%) deaths. There were no differences in average perfusion characteristics between treatment arms. Analyzing the relationship between microcirculatory perfusion and mortality, we found an association between vascular density parameters and mortality. Total vascular density (beta $=0.006, p<0.003$ ), perfused vascular density (beta $=0.005, p<$ 0.04 ), and De Backer score (beta $=0.009, p<0.01$ ) were higher overall in survivors in a generalized estimating equation model, and this association was significant at the 72-h time point ( $p<0.05$ for each parameter).

Conclusions: Microcirculatory perfusion did not differ between three early septic shock treatment arms. We found an association between microcirculatory perfusion parameters of vascular density at $72 \mathrm{~h}$ and mortality.

Trial registration: ClinicalTrials.gov, NCT00510835. Registered on August 2, 2007.

Keywords: Sepsis, Microcirculation, Pathophysiology, Mortality

\section{Background}

Patients with sepsis have high morbidity, mortality, and care costs. Improving outcomes requires an enhanced understanding of the complex pathophysiology of the disease. Organ dysfunction and multisystem organ failure are common precursors to death in sepsis. The smallest blood vessels of the microcirculation $(<20 \mu \mathrm{m}$ in diameter) are the principal sites of gas and nutrient exchange between blood and underlying tissues [1].

\footnotetext{
* Correspondence: nshapiro@bidmc.harvard.edu

'Department of Emergency Medicine and Center for Vascular Biology

Research, Beth Israel Deaconess Medical Center, 1 Deaconess Road, CC2-W,

Boston, MA 02215, USA

Full list of author information is available at the end of the article
}

Microcirculatory perfusion disturbances represent a direct physiologic link to multisystem organ dysfunction. Microcirculatory perfusion disturbances represent a potential universal link across organs and could alter insights and care.

Possible causes of microcirculatory perfusion alterations in sepsis include endothelial cell dysfunction, glycocalyx degradation, increased leukocyte adhesion, microthrombus formation, rheological abnormalities, altered local perfusion pressures due to regional redistribution of blood flow, and functional shunting. The microcirculation is impaired in sepsis [2-14], and the defect in perfusion may be therapeutically reversed $[4$, 15-17]. With the advent of handheld in vivo imaging

(C) The Author(s). 2018 Open Access This article is distributed under the terms of the Creative Commons Attribution 4.0 International License (http://creativecommons.org/licenses/by/4.0/), which permits unrestricted use, distribution, and 
modalities, it is possible to visualize the sublingual microcirculation in human patients at the bedside [1, 18-20].

Microcirculatory perfusion represents the combination of the density of vessels available to provide nutrients (e.g., oxygen) carrying blood to the organs and the flow rates of that blood. Clinical studies suggest that persistent microcirculatory alterations refractory to resuscitation are prognostic of fatal outcome $[3,4,8-10,12-15$, $17,21,22]$ independent of systemic variables and oxygen-derived variables $[4,8,17]$. Changes in microvascular perfusion may occur in the absence of global hemodynamic perturbations (i.e., low blood pressure/ cardiac output), indicating that these alterations are intrinsic to the microcirculation.

Prior studies of microcirculatory perfusion disturbances in sepsis and septic shock have typically been either limited in size $[3,8,10,14,17,23]$ or initiated in the intensive care unit (ICU) well after the onset of sepsis. For this project, we studied patients enrolled in the Protocolized Care for Early Septic Shock (ProCESS) study, a randomized clinical trial of three alternative resuscitation strategies that included the administration of fluids, vasopressors, blood, and dobutamine (each with a previously published impact on the microcirculation). We sought to (1) determine the effects of alternative resuscitation strategies on microcirculatory perfusion disturbances in early septic shock and (2) study the association between microcirculatory perfusion disturbances over the first $72 \mathrm{~h}$ of resuscitation and 60-day in-hospital mortality in early septic shock.

\section{Methods}

\section{Study aim, design, and setting design}

We enrolled a subpopulation of subjects participating in the ProCESS trial, a patient-level randomized multicenter interventional trial of alternative resuscitation strategies in emergency department (ED) early septic shock [24]. In the ProCESS trial, subjects with sepsis and hypoperfusion (see enrollment criteria below) randomly received one of three resuscitation strategies: early goal-directed therapy (EGDT) as described by Rivers et al. [25] and delivered by a study team, a strategy of noninvasive protocolized care delivered by a study team, or usual care absent any protocol or prompts and delivered by the clinical team [24]. Six hospital sites participated in this ancillary study to the ProCESS trial.

The primary outcome was in-hospital mortality by day 60. We registered the current trial and ProCESS with ClinicalTrials.gov under the identifiers NCT00793442 and NCT00510835, respectively, and the Beth Israel Deaconess Medical Center Committee for Clinical Investigations and each site's institutional review board approved the design. Each subject or legal representative gave written informed consent.

\section{Participants}

ProCESS trial subjects [24] all had (1) suspected infection in the ED; (2) at least two systemic inflammatory response syndrome criteria [26]; and (3) refractory hypotension, defined as a systolic blood pressure $<$ $90 \mathrm{mmHg}$ despite an intravenous fluid challenge of at least $1 \mathrm{~L}$ of crystalloids or evidence of tissue hypoperfusion (blood lactate concentration $\geq 4 \mathrm{mmol} / \mathrm{L}$ ). They were enrolled as a convenience sample at sites participating in this ancillary study. An additional exclusion criterion for this specific ancillary study was the inability to tolerate study procedures due to an oxygen requirement; for example, a patient on a nonrebreather mask could not tolerate removing the mask to perform the microcirculation image collection.

\section{Demographics and clinical data collection}

We collected information on patient demographics, comorbid illnesses, etiologies of infection, and treatments. We also collected macrocirculatory perfusion parameters.

\section{Microcirculatory video microscopy image capture and management}

We visualized and recorded the sublingual microcirculation using sidestream dark field video microscopic imaging (MicroScan; MicroVision Medical, Inc., Amsterdam, The Netherlands) as previously described $[18,20,27]$. We captured videos at 6,24 , and $72 \mathrm{~h}$ after enrollment. We specifically did not attempt microcirculation imaging during the initial $6-\mathrm{h}$ resuscitation period so as not to affect the main trial's intervention. We uploaded stored video clips to the central laboratory using both a proprietary file transfer software (Studymaker File Manager; Studymaker, Newton, MA, USA) and a cloud storage service (Dropbox; Dropbox, Inc., San Francisco, CA, USA) [18, 27, 28].

\section{Site training}

Study team participants received instruction on the operation and use of the MicroScan device primarily through live training during individualized training sessions provided at their institution. Instruction consisted of (1) overview of the pathophysiology of microcirculatory derangements in sepsis; (2) introduction to the MicroScan device as well as its setup and operation; and (3) a detailed presentation of the technique for image acquisition, including positioning, lighting, focus, and image recording. All participants then practiced using healthy volunteers. We focused on the five points for image acquisition outlined by the microcirculation consensus conference as key for quality image analysis: (1) obtain five sample sites per subject, (2) avoid pressure artifacts, (3) eliminate secretions, (4) adequate focus and brightness adjustment, and (5) recording quality [29]. 
Study team members were required to demonstrate proficiency in obtaining images by adequately recording a video to "pass" training.

\section{Image analysis}

We processed MicroScan video files to enhance contrast, edited to visually stable sequences of 3-10 s in duration, and evaluated for image quality using the method described by Massey et al. [27]. We sorted video clips using an ordinal quality score with up to three clips selected at each of the three time points, and then we assigned a random identifier before further microvascular analysis using AVA 3.1 software (MicroVision Medical BV). We did not rely on automated vessel identification; instead, vessel centerlines and lumen boundaries were drawn using manual tools in AVA. Vessel classification was done using sizing, with small, medium, and large vessels having lumen diameters < $20 \mu \mathrm{m}, 20-50 \mu \mathrm{m}$, and $>50 \mu \mathrm{m}$, respectively. All of the microcirculatory parameters reported were from analyses of small vessels $(<20 \mu \mathrm{m})$ because capillaries are the principal sites of oxygen exchange and fall into the small vessel size range.

\section{Scoring the images}

For microcirculatory perfusion image analysis, we followed the methods of the 2007 consensus conference on evaluation of the microcirculation [29]. We report the suggested measures of proportion of perfused vessels (PPV), microcirculatory flow index (MFI), De Backer score, total vascular density (TVD), perfused vascular density (PVD), and heterogeneity index (Table 1) [29]. PPV is the perfused linear density of small vessels in a field of view, computed as the length of perfused small vessels divided by the total length of small vessels visualized $[18,30]$. MFI calculations followed the semiquantitative technique described by Spronk et al. [15] ( 0 = absent flow; 1 = sludging/noncontinuous flow; $2=$ moderate flow; $3=$ normal $/$ brisk flow), determining the mode for each quadrant and averaging over the four quadrants to yield the MFI. De Backer score calculations used the line crossing technique [31]. We calculated TVD by quantifying the total density of small vessels within the field of view and calculated PVD by measuring the density of perfused small vessels within the field of view. Heterogeneity index calculations used the highest MFI over the four quadrants, subtracting the lowest MFI over the four quadrants and dividing by the mean MFI $[8,29]$. For the perfusion parameters, we considered any vessel segment with a flow score greater than or equal to 2 (moderate/normal flow) as perfused. After vessel detection, we used the AVA software to make the calculations described above and quantify vessel length. We calculated the PPV by dividing the total vessel length by the perfused vessel length.

\section{Statistical analysis}

We assessed for normality and proceeded with parametric or nonparametric testing, with two-tailed alpha set at 0.05. The microcirculatory perfusion parameters of interest were PPV, MFI, De Backer score, TVD, PVD, and heterogeneity index. For any effect of the randomly assigned treatment strategies on the different microcirculatory perfusion parameters, we report the mean (plus $\mathrm{SD})$ or median [IQR] for each of the microcirculatory perfusion parameters by study arm. For differences in microcirculatory perfusion parameters by mortality endpoint, we report the differences in demographics, comorbidities, and sepsis etiologies between survivors and nonsurvivors.

For the analysis of an association between assigned treatment arm and microcirculation parameters, we used

Table 1 Summary of microcirculatory parameters quantitative metrics

\begin{tabular}{|c|c|c|}
\hline Name & Abbreviation & Description \\
\hline $\begin{array}{l}\text { Microcirculatory flow } \\
\text { index }\end{array}$ & MFI & $\begin{array}{l}\text { A qualitative assessment of flow over quadrants. Predominant (mode) flow velocity of visualized vessels is } \\
\text { determined for each image quadrant. MFI is computed as the average of the predominant flow classification } \\
\text { of small vessels over the four image quadrants. }\end{array}$ \\
\hline Heterogeneity index & HETERO & $\begin{array}{l}\text { A measure of flow heterogeneity. Computed as the maximum MFI quadrant value minus minimum MFI } \\
\text { quadrant value divided by mean MFI. }\end{array}$ \\
\hline Total vascular density & TVD & $\begin{array}{l}\text { A measure of all vessels over the field of view. Computed as total length of small vessels divided by total area } \\
\text { of field of view. }\end{array}$ \\
\hline $\begin{array}{l}\text { Proportion of } \\
\text { perfused vessels }\end{array}$ & PPV & $\begin{array}{l}\text { A measure of the lineal proportion of perfused vessels. Computed as total length of perfused small vessels } \\
\text { (semiqualitative velocity score } 2 \text { or greater) }{ }^{a} \text { divided by total length of small vessels. }\end{array}$ \\
\hline $\begin{array}{l}\text { Perfused vascular } \\
\text { density }\end{array}$ & PVD & $\begin{array}{l}\text { A measure of the perfused vessel lineal density. Computed as proportion of perfused vessels multiplied by } \\
\text { total vessel density. }\end{array}$ \\
\hline De Backer score & De Backer & $\begin{array}{l}\text { An alternate measure of total vessel density. A grid is formed using three equally spaced vertical and three } \\
\text { horizontal lines over image area. Computed as number of vessels crossing grid lines divided by the total } \\
\text { length of the lines. }\end{array}$ \\
\hline
\end{tabular}

a Semiquantitative velocity scoring: $0=$ absent flow; $1=$ noncontinuous/sluggish flow; $2=$ moderate flow; $3=$ normal continuous/brisk flow [15, 29]

A summary of microcirculatory flow metrics following the recommendations of a consensus report [29] 
a generalized estimating equation (GEE) with a subject being a cluster (nonstructured correlation matrix) with pairwise comparisons with adjustment for multiple comparisons (LSD). For the normally distributed parameters of TVD, PVD, and De Backer score, we used linear models within GEE; for MFI and PPV, which were skewed, we applied a gamma distribution with log-link functions. For the heterogeneity index parameter, we chose a Tweedie distribution. Within each model, we assessed the interaction between the microcirculation parameter and time point. Because the interaction between the microcirculation parameters and time was not significant in all models, we report the results based on the models without the interactions included. For the analysis of the association between microcirculation parameters and 60-day in-hospital mortality, we used GEE with subject being a cluster (nonstructured correlation matrix) using logistic regression with robust estimator and unstructured correlation matrix adjusted for Charlson comorbidity index and age.

Finally, we compared the mean value of macrocirculatory parameters among survivors and nonsurvivors. We used GEE models to evaluate the association between the macrocirculatory and microcirculatory parameters adjusted for age and Charlson comorbidity index score.

\section{Sample size}

To estimate sample size for the impact of the resuscitation protocols on microcirculatory perfusion based on pilot data, we used an estimated mean of MFI (considered the "main" microcirculatory perfusion parameter reported at the time) at $2.3 \pm 0.6$ for standard care, expecting subjects with protocolized goal-directed resuscitation to have a mean MFI 20\% greater than this mean. At a power of 0.9 to detect a mean difference of 0.46 , the estimated sample size was 114. For the association between MFI and mortality, we estimated that the OR of mortality would increase by $50 \%$ per 0.5 of an SD decrease in the MFI (thus an OR of 1.5 for a 0.5 -SD decrease). For type I error of 0.05 and a power of 0.9 , the estimated sample size was 115 subjects. Because the current reported trial was ongoing and part of a larger parent trial collecting biomarkers for up to 600 patients, in consultation with the data and safety monitoring board, we continued enrollment to surpass our sample size estimates to increase our overall power.

\section{Results}

From the parent trial of 1341 patients, we enrolled 225 (16.8\%) into this substudy, and 207 (92\%) subjects had adequate images and were analyzed. A total of $1244 \mathrm{im}$ ages from 439 time points were included (an average of 2.8 videos per patient time point).

\section{Patient demographics}

Our study subjects had a mean age of 61 years (SD 16), $73 \%$ were white, and $20 \%$ were African American (Table 2). Similar to prior studies, the prevalence of comorbid illness was high, including high rates of hypertension, diabetes, and cancer. Pneumonia was the most common underlying etiology of sepsis. The baseline Sequential Organ Failure Assessment score was 7.7 (SD 3.8). Among the 207 participants, there were 40 deaths, for an overall mortality rate of $19.3 \%$. The population enrolled in this ancillary study was very similar to and representative of the population enrolled in the ProCESS study overall (Additional file 1: Table S1).

\section{Microcirculatory perfusion image analysis}

There were 225 total patients with images obtained across the 6-, 24-, and 72-h time points, with attempts made during $552(86 \%)$ of the available time points (Additional file 2: Table S2). Among the images processed and analyzed, 439 (80\%) of the time points had images of suitable quality for analysis. The success of image acquisition by time point was as follows: $6 \mathrm{~h}$ (170 of $205 ; 83 \%), 24 \mathrm{~h}$ (149 of 200; 75\%), and $72 \mathrm{~h}$ (120 of $147 ; 82 \%)$. Pressure artifacts were the most common reason for not passing our quality check ( $40 \%$ of failures), followed by content artifacts ( $30 \%$ of failures).

\section{Effect of resuscitation strategy on microcirculatory perfusion}

There were no differences in clinical outcome between resuscitation strategies in the parent ProCESS trial [24]. Similarly, there were no differences in the microcirculatory perfusion parameters of PPV, De Backer score, TVD, PVD, or heterogeneity index for each time point between the three treatment arms (Table 3 and Additional file 3: Table S3). MFI did show a difference between the three arms with impaired perfusion in the EGDT group; however, the mean difference was quite small, which limits clinical impact. Because there were no meaningful differences in microcirculatory perfusion patterns between the groups, the treatment groups were pooled for subsequent analyses.

\section{Association between microcirculatory perfusion parameters and mortality}

The microcirculation parameters of TVD (beta $=0.006$, $p<0.003$ ), PVD (beta $=0.005, p<0.04$ ), and De Backer (beta $=0.009, p<0.01$ ) scores were higher in survivors in a GEE model that considered each parameter at all time points (Table 4 and Fig. 1). This indicates an impairment of the microcirculation in those who eventually died. These differences were driven primarily by the $72-\mathrm{h}$ TVD, PVD, and De Backer scores, which were lower among nonsurvivors at $72 \mathrm{~h}$. The microcirculation 
Table 2 Demographics

\begin{tabular}{|c|c|c|c|}
\hline Characteristic & Entire cohort $(N=207)$ & Lived $(n=167)$ & Died $(n=40)$ \\
\hline Age, years, mean (SD) & $60.9(15.6)$ & $59.44(15.95)$ & $67.23(12.37)$ \\
\hline Female sex & $106(51)$ & $87(52)$ & $22(55)$ \\
\hline \multicolumn{4}{|l|}{ Race } \\
\hline White & $152(73)$ & $124(74)$ & $28(70)$ \\
\hline Black or African American & $41(20)$ & $31(19)$ & $10(25)$ \\
\hline Asian & $8(3.9)$ & $7(4.2)$ & $1(2.5)$ \\
\hline Other & $5(2.4)$ & $4(2.4)$ & $1(2.5)$ \\
\hline \multicolumn{4}{|l|}{ Ethnicity } \\
\hline Non-Hispanic & $189(91)$ & $149(89)$ & $40(100)$ \\
\hline Hispanic & $18(8.7)$ & $18(11)$ & $0(0.00)$ \\
\hline \multicolumn{4}{|l|}{ Domicile prior to admission } \\
\hline Non-nursing home & $185(89)$ & $149(89)$ & $36(90)$ \\
\hline Nursing home & $22(11)$ & $18(11)$ & $4(10)$ \\
\hline \multicolumn{4}{|l|}{ Chronic conditions } \\
\hline Charlson comorbidity index score, mean (SD) & $3.1(2.7)$ & $2.77(2.5)$ & $4.35(3.3)$ \\
\hline Hypertension & $123(59)$ & $95(57)$ & $28(70)$ \\
\hline Diabetes mellitus & $64(31)$ & $48(29)$ & $16(40)$ \\
\hline Chronic respiratory disease & $54(26)$ & $43(26)$ & $11(28)$ \\
\hline Cancer & $52(25)$ & $38(23)$ & $14(35)$ \\
\hline Dialysis-dependent renal impairment & $14(6.8)$ & $12(7.1)$ & $2(5.0)$ \\
\hline Congestive heart failure & $29(14)$ & $22(13)$ & $7(18)$ \\
\hline Prior myocardial infarction & $22(11)$ & $15(9.0)$ & $7(18)$ \\
\hline Cerebral vascular disease & $21(10)$ & $17(10)$ & $4(10)$ \\
\hline Peripheral vascular disease & $21(10)$ & $14(8.4)$ & $7(18)$ \\
\hline Chronic dementia & $14(6.8)$ & $10(6.0)$ & $4(10)$ \\
\hline Hepatic cirrhosis & $17(8.2)$ & $11(6.6)$ & $6(15)$ \\
\hline Peptic ulcer disease & $11(5.3)$ & $9(5.4)$ & $2(5.0)$ \\
\hline AIDS and related syndromes & $1(1.9)$ & $2(1.2)$ & $2(5.0)$ \\
\hline \multicolumn{4}{|l|}{ Source of sepsis } \\
\hline Pneumonia & 69 (33.3) & 57 (34.) & $12(30)$ \\
\hline Urosepsis & $40(19.3)$ & $32(19)$ & $8(20)$ \\
\hline Infected, source unknown & $19(9.2)$ & $16(9.6)$ & $3(7.5)$ \\
\hline Intra-abdominal infection & $33(16)$ & $22(13)$ & $11(28)$ \\
\hline Skin and soft tissue infections & $14(6.8)$ & $13(7.8)$ & $1(2.5)$ \\
\hline Catheter-related infection & $14(6.8)$ & $11(6.6)$ & $3(7.5)$ \\
\hline Central nervous system & $2(1.0)$ & $2(1.2)$ & $0(0.0)$ \\
\hline Endocarditis & $4(1.9)$ & $4(2.4)$ & $0(0.0)$ \\
\hline Other & $9(4.4)$ & $8(4.8)$ & $1(2.5)$ \\
\hline Considered after review not to be infected & $3(1.5)$ & $2(1.2)$ & $1(2.5)$ \\
\hline Baseline SOFA score, mean (SD) & $7.7(3.8)$ & $6.96(3.4)$ & $10.83(3.8)$ \\
\hline
\end{tabular}

AIDS Acquired immunodeficiency syndrome, SOFA Sequential Organ Failure Assessment Data are number (\%) unless otherwise noted 
Table 3 Analysis of an association between arms and microcirculation parameters

\begin{tabular}{|c|c|c|c|c|}
\hline & \multicolumn{2}{|c|}{ EGDT vs. control } & \multicolumn{2}{|c|}{ Noninvasive vs. control } \\
\hline & Beta & $p$ Value & Beta & $p$ Value \\
\hline Total vascular density $\left(\mathrm{mm} / \mathrm{mm}^{2}\right)$ & -0.92 & 0.16 & 0.23 & 0.72 \\
\hline Perfused vascular density $\left(\mathrm{mm} / \mathrm{mm}^{2}\right)$ & -1.29 & 0.07 & -0.24 & 0.74 \\
\hline De Backer score & -0.42 & 0.30 & 0.05 & 0.91 \\
\hline Heterogeneity index & 0.11 & 0.09 & 0.02 & 0.71 \\
\hline Microcirculatory flow index & -0.06 & $<0.02$ & -0.02 & 0.36 \\
\hline Proportion perfused vessels (\%) & -0.05 & 0.09 & 0.00 & 0.94 \\
\hline
\end{tabular}

EGDT Early goal-directed therapy

In this analysis, a generalized estimating equation model adjusted for age and Charlson comorbidity index score was used to assess differences in microcirculatory flow parameters between treatment arms. The only parameter found to be statistically significant was microcirculatory flow index, which was lower in the early goal-directed therapy group compared with control; however, the small difference limits the clinical significance of this finding. There was no significant difference across all other parameters when comparing each of the arms

Boldface indicates $p<0.05$

parameters of MFI, PPV, and heterogeneity index were similar among survivors and nonsurvivors at each time point and in the overall models (Table 4 and Fig. 1).

\section{Association between macrocirculatory and microcirculatory parameters}

In an exploratory analysis, we examined the relationship between macrocirculatory parameters and microcirculatory parameters. First, we assessed differences in macrocirculatory and microcirculatory parameters among survivors and nonsurvivors in aggregate (Table 5) and found survivors to have a higher driving pressure (MAP - CVP) and lower serum lactate, whereas there were no differences in the other parameters. In an adjusted analysis, only increased CVP was associated with higher TVD, De Backer score, and heterogeneity index, whereas there was an unexpected correlation between CVP and lower MFI score as well as between mean arterial pressure and lower TVD (Table 6). We identified a weak correlation between CVP and some flow (heterogeneity index) and density parameters (TVD and De Backer score); however, overall, the data do not show a meaningful association between macrocirculatory and microcirculatory parameters.

\section{Discussion}

Prior studies have shown a relationship between early microcirculatory indices and survival during the resuscitation phase of therapy [3, 4, 8, 22]. De Backer et al. [22] found that decreased microcirculatory perfusion as measured by PPV, PVD, and MFI was associated with mortality. They reported that the PPV parameter was the strongest predictor of mortality and that this association was maintained in multiple logistic regression models for both early $(<24 \mathrm{~h})$ and late $(\geq 24 \mathrm{~h})$ time points. The overall AUCs for mortality for PPV and PVD were 0.82 and 0.74 , respectively. In a similar study, Trzeciak et al. [8] investigated 26 patients with sepsis in the ED and

Table 4 Differences in microcirculation parameters between survivors and nonsurvivors

\begin{tabular}{|c|c|c|c|c|c|c|c|c|}
\hline \multirow[t]{2}{*}{ Microcirculation parameter } & \multicolumn{3}{|c|}{ Alive at discharge } & \multicolumn{3}{|c|}{ Dead at discharge } & \multicolumn{2}{|c|}{$\begin{array}{l}\text { Overall model } \\
\text { Alive vs. dead }\end{array}$} \\
\hline & $\begin{array}{l}6 \mathrm{~h} \\
n=138\end{array}$ & $\begin{array}{l}24 h \\
n=128\end{array}$ & $\begin{array}{l}72 \mathrm{~h} \\
n=108\end{array}$ & $\begin{array}{l}6 h \\
n=32\end{array}$ & $\begin{array}{l}24 \mathrm{~h} \\
n=21\end{array}$ & $\begin{array}{l}72 \mathrm{~h} \\
\mathrm{n}=12\end{array}$ & Beta & $\begin{array}{l}p \\
\text { Value }^{*}\end{array}$ \\
\hline $\mathrm{TVD}$, mean $\pm \mathrm{SD}$ & $22.6 \pm 4.2$ & $23.0 \pm 5.4$ & $22.1 \pm 4.0$ & $21.1 \pm 3.7$ & $21.7 \pm 5.7$ & $19.3 \pm 5.1$ & 0.006 & $<0.003$ \\
\hline$P V D$, mean $\pm S D$ & $21.1 \pm 4.8$ & $21.8 \pm 5.7$ & $21.0 \pm 4.2$ & $20.8 \pm 4.9$ & $19.3 \pm 7.5$ & $17.6 \pm 4.8$ & 0.005 & $<0.04$ \\
\hline De Backer score, mean \pm SD & $14.8 \pm 2.6$ & $15.1 \pm 3.2$ & $14.6 \pm 2.5$ & $14.5 \pm 2.6$ & $14.4 \pm 2.3$ & $12.5 \pm 3.7$ & 0.009 & $<0.01$ \\
\hline $\begin{array}{l}\text { Heterogeneity index, median } \\
\text { [IQR] }\end{array}$ & $\begin{array}{l}0.08[0.00- \\
0.43]\end{array}$ & $\begin{array}{l}0.18[0.00- \\
0.46]\end{array}$ & $\begin{array}{l}0.17[0.00- \\
0.49]\end{array}$ & $\begin{array}{l}0.00[0.00- \\
0.51]\end{array}$ & $\begin{array}{l}0.27[0.00- \\
0.67]\end{array}$ & $\begin{array}{l}0.29[0.00- \\
0.60]\end{array}$ & $\overline{-} 0$. & 0.54 \\
\hline MFI, median [IQR] & $\begin{array}{l}2.92[2.59- \\
3.00]\end{array}$ & $\begin{array}{l}2.85[2.61- \\
3.00]\end{array}$ & $\begin{array}{l}2.88[2.51- \\
3.00]\end{array}$ & $\begin{array}{l}2.96[2.56- \\
3.00]\end{array}$ & $\begin{array}{l}2.67[2.18- \\
3.00]\end{array}$ & $\begin{array}{l}2.71[2.25- \\
3.00]\end{array}$ & 0.05 & 0.33 \\
\hline PPV, median [IQR] & $\begin{array}{l}0.90[0.83- \\
0.97]\end{array}$ & $\begin{array}{l}0.90[0.85- \\
0.96]\end{array}$ & $\begin{array}{l}0.91[0.83- \\
0.95]\end{array}$ & $\begin{array}{l}0.91[0.82- \\
0.95]\end{array}$ & $\begin{array}{l}0.91[0.83- \\
0.95]\end{array}$ & $\begin{array}{l}0.92[0.69- \\
1.00]\end{array}$ & $\begin{array}{l}- \\
1.16\end{array}$ & 0.46 \\
\hline
\end{tabular}

Abbreviations: MFI Microcirculatory flow index, PPV Proportion of perfused vessels, PVD Perfused vascular density, TVD Total vascular density *In bold, $p<0.05$ for pairwise comparisons at a given time point

The mean $( \pm S D)$ and median $[I Q R]$ values are shown for each microcirculation parameter at 6-, 24-, and 72-h time points for survivors and nonsurvivors. TVD, PVD, and De Backer scores are lower in nonsurvivors, indicating an impaired microcirculation. In generalized estimating equation models, TVD, PVD, and De Backer scores were lower overall in nonsurvivors than in survivors 


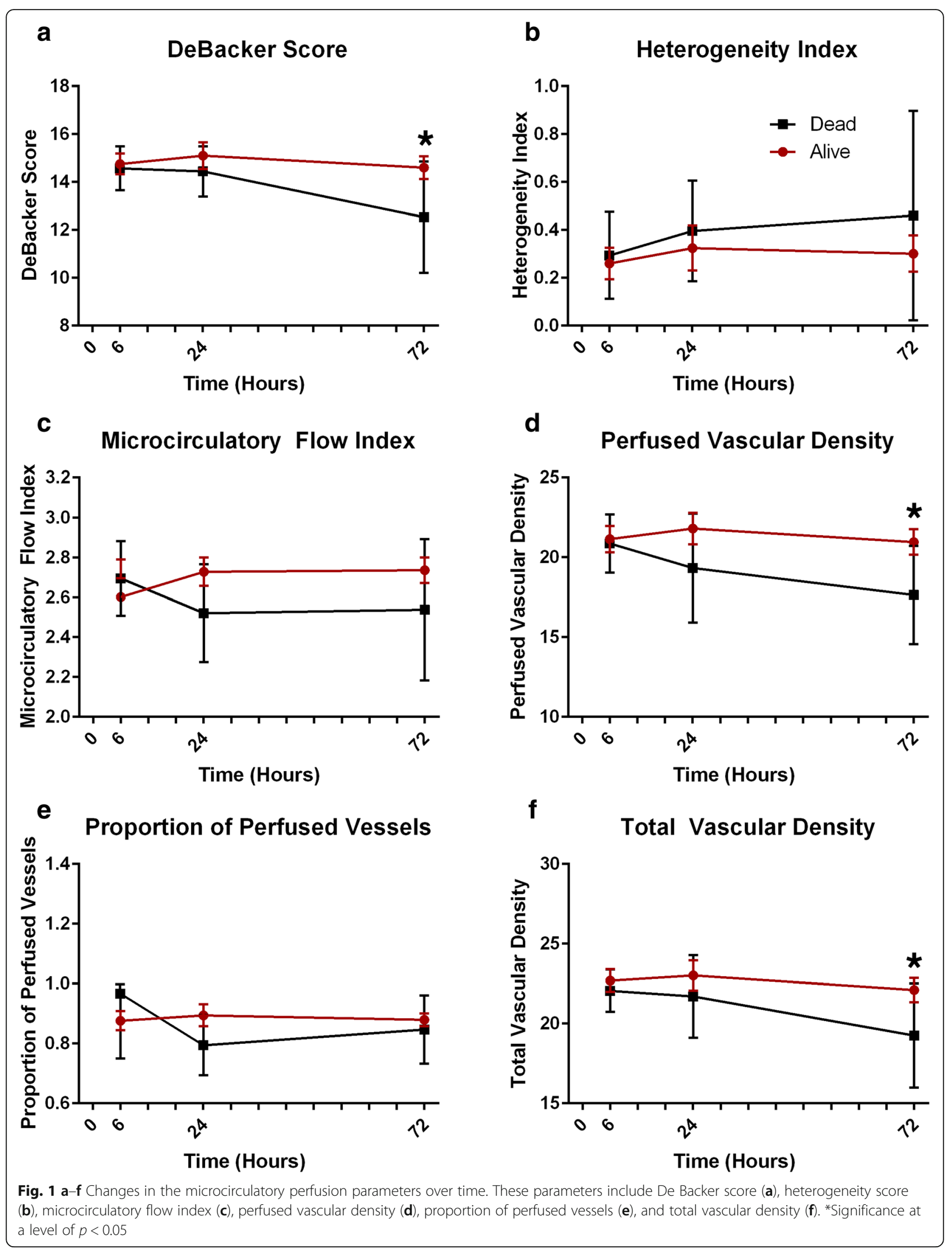


Table 5 Average macrocirculatory parameters among survivors and nonsurvivors

\begin{tabular}{llll}
\hline & $\begin{array}{l}\text { Dead } \\
n=40\end{array}$ & $\begin{array}{l}\text { Alive } \\
n=167\end{array}$ & $p$ Value \\
\hline Systolic blood pressure $(\mathrm{mmHg})$ & $104.9 \pm 14.3$ & $108.7 \pm 14.9$ & 0.20 \\
Diastolic blood pressure $(\mathrm{mmHg})$ & $56.8 \pm 9.5$ & $59.3 \pm 8.4$ & 0.14 \\
Mean arterial pressure $(\mathrm{mmHg})$ & $72.8 \pm 9.5$ & $75.8 \pm 9.3$ & 0.11 \\
Heart rate (beats per minute) & $98.2 \pm 19.1$ & $94.3 \pm 17.7$ & 0.27 \\
Central venous pressure $(\mathrm{mmHg})$ & $14.0 \pm 5.3$ & $11.8 \pm 5.2$ & 0.11 \\
Scvo $_{2}$ & $76.9 \pm 6.1$ & $73.4 \pm 10.7$ & 0.32 \\
Driving pressure (mmHg) & $56.1 \pm 5.4$ & $63.2 \pm 9.7$ & 0.004 \\
Lactate (mmol/dl) & $4.7 \pm 4.5$ & $1.7 \pm 1.2$ & $<0.001$ \\
\hline
\end{tabular}

$\mathrm{SCVO}_{2}$ Central venous oxygen saturation

Table shows the average macrocirculatory flow parameters based on survival status incorporating multiple time points

found that impaired flow and increased heterogeneity of flow were significantly disturbed features of the microcirculation in nonsurvivors compared with survivors. Furthermore, in a study of 49 ICU patients in septic shock, Sakr et al. [4] found that there was no difference in microcirculatory perfusion parameters at the onset of shock, but survivors were able to restore their microcirculatory perfusion as indicated by significant differences in PPV, whereas nonsurvivors had persistently impaired perfusion.

Our findings support a role for microcirculatory perfusion disturbances in sepsis pathophysiology. However, we found a variable association between the different microcirculatory perfusion parameters and mortality. Our study approach and findings support the Sakr et al. [4] study findings, where the initial adequacy of microcirculatory perfusion was not as important as the ability to recover microcirculatory perfusion over time to predict mortality. Our study also demonstrated that parameters of vessel density (TVD and De Backer score) and density of vessels with flow (PVD) were more highly associated with outcome than parameters of flow alone, such as our primary outcome of MFI, or PPV, in which there were not significant differences. Our study occurred during a clinical trial of resuscitation therapy in which patients were treated with a structured resuscitation strategy or with usual care that was aggressive but different from the structured approaches, and the latter performed in a similar fashion for most outcomes. This usual care in the parent trial may differ from previous "wild-type" treatments in observational trials. Although our study supports an association between impaired microcirculatory perfusion and mortality in sepsis, our findings suggest that this association is perhaps less robust than others have suggested.

As highlighted above, there is no clear consensus on which microcirculatory perfusion parameter is most important. For example, De Backer et al. previously found PPV to be the parameter most strongly associated with mortality [3, 22]. In this study, we found the measures of density, namely TVD, PVD, and De Backer score (an estimate of total density), to be associated with mortality when considering all parameters at all time points in a single model and at the 72-h time period. Sakr et al. [4] found PPV to be the most prognostic of outcome [18]. Although the parameters PPV (based on per-vessel quality of flow) and MFI (based on a visual estimate of overall flow quality) have previously been demonstrated to be the most important predictors of outcome, our results support that the density parameters of TVD, PVD, and De Backer score were more tightly associated with mortality and thus perhaps more important. On the other hand, the ability of blood to deliver oxygen is paramount; thus, presence of circulating red blood cells may be more important than the speed at which they are flowing.

There are a number of limitations to this study. First, there is a potential selection bias because we obtained videos only in a subset of subjects; thus, it is possible that those who were more (or less) ill may have had successful image acquisition in a nonrandom fashion.

Table 6 Relationship between macrocirculatory and microcirculatory parameters

\begin{tabular}{llllllll}
\hline & MAP & SBP & DBP & Heart rate & CVP & MAP - CVP & Lactate \\
\hline TVD $\left(\mathrm{mm} / \mathrm{mm}^{2}\right)$ & $\mathbf{- 0 . 0 3 8 ( \mathbf { 0 . 0 1 8 } )}$ & $-0.076(0.34)$ & $0.075(0.30)$ & $-0.01(0.396)$ & $\mathbf{0 . 3 2}(\mathbf{0 . 0 3 1})$ & $-0.15(0.17)$ & $-0.071(0.59)$ \\
PVD small $\left(\mathrm{mm} / \mathrm{mm}^{2}\right)$ & $0.022(0.61)$ & $0.032(0.26)$ & $-0.004(0.93)$ & $-0.005(0.738)$ & $-0.011(0.91)$ & $0.024(0.56)$ & $-0.457(0.07)$ \\
De Backer score & $-0.034(0.75)$ & $-0.032(0.58)$ & $-0.023(0.83)$ & $-0.002(767)$ & $\mathbf{0 . 2 3}(\mathbf{0 . 0 4 8})$ & $-0.065(0.47)$ & $-0.188(0.27)$ \\
Heterogeneity index & $-0.001(0.92)$ & $-0.004(0.59)$ & $\mathbf{0 . 1 6 ( < 0 . 0 0 1 )}$ & $\mathbf{- 0 . 0 1 2 ( \mathbf { 0 . 0 2 } )}$ & $\mathbf{0 . 0 5 1 ( \mathbf { 0 . 0 1 5 } )}$ & $-0.01(0.40)$ & $0.004(0.91)$ \\
MFI & $0.00(0.89)$ & $0.00(0.68)$ & $0.00(0.91)$ & $0.001(0.738)$ & $\mathbf{- 0 . 0 2 8 ( < 0 . 0 0 1 )}$ & $0.003(0.075)$ & $-0.013(0.057)$ \\
PPV (\%) & $0.001(0.75)$ & $0.00(0.75)$ & $0.00(0.79)$ & $0.001(0.301)$ & $-0.005(0.11)$ & $0.001(0.39)$ & $-0.022(0.24)$ \\
\hline
\end{tabular}

Abbreviations: MAP Mean arterial pressure, SBP Systolic blood pressure, DBP Diastolic blood pressure, CVP Central venous pressure, TVD Total vascular density, PVD Perfused vascular density, MFI Microcirculatory flow index, PPV Proportion of perfused vessels

We used generalized estimating equation models to evaluate the association between the macrocirculatory and microcirculatory parameters adjusted for age and Charlson score. For TVD, PVD, and De Backer scores that were normally distributed, we used linear models as link functions; for MFI, PPV due to the skewness, we applied gamma distribution with log-link functions and for heterogeneity index, Tweedie distribution was chosen due to the abundance of observations at 0 . Beta with ( $p$ value) is shown Boldface indicates $p<0.05$ 
However, our ancillary study cohort with successful image acquisition did have a mortality rate similar to that in the overall trial. Second, there were a number of trained operators across centers but with varying prior experience with microcirculatory flow image acquisition; it is possible that suboptimal image acquisition influenced the results (e.g., pressure artifact; if the operator pushed down too hard, it may have given the false perception of occluded flow). We tried to guard against this through an approach of selecting and including only videos judged as free of such influences. Conversely, our exclusion of a relatively high ratio of videos with presumed pressure artifacts may have contributed to bias. It is possible that pressure artifacts were more likely in patients with more (or less) severe illness. Third, to avoid affecting the ProCESS intervention, we delayed our image acquisition until after the experimental protocol intervention period (the first $6 \mathrm{~h}$ after eligibility) was over; it is possible that microcirculation could have differed at baseline. Fourth, other factors, such as chronic diseases or other confounders, may have altered the association of microcirculatory perfusion parameters with mortality. Fifth, we assessed multiple microcirculatory flow and density parameters simultaneously, and it is possible that some significant results were type I errors. Finally, we identified vessels in the images by eye, drew them by hand, and visually estimated the flow rate. It is possible that other automated techniques may yield different results.

Future initiatives should continue to focus on delineating which microcirculatory parameters have the most significant pathophysiologic impact. We found that measures of microvessel density (TVD and De Backer score) and perfusion (PVD) were associated with mortality, whereas measures of flow quality were not. Similarly, software development for analysis, especially at the point of care, may aid future efforts. We used a semiquantitative technique whereby we traced vessels by hand and made empiric estimates on the rate of flow. Reliable automated techniques for vessel identification and flow assessment are still needed.

\section{Conclusions}

We found that the microcirculation in patients in septic shock was not differentially influenced by these three early treatment strategies, which included two protocolized approaches guided by specific physiologic input. There was not an association between microcirculatory perfusion parameters; however, we observed a positive association between microvascular density parameters measured at $72 \mathrm{~h}$ and in-hospital mortality by day 60 . Conventional resuscitation therapy incompletely normalized microcirculatory perfusion in nonsurvivors. Novel agents that target the restoration of microcirculatory perfusion disturbances may be a promising future therapeutic approach in sepsis.

\section{Additional files}

\begin{abstract}
Additional file 1: Table S1. The overall ProCESS population compared with the population enrolled in the microcirculatory flow ancillary study. * $p<0.05$. (DOCX $17 \mathrm{~kb}$ )

Additional file 2: Table S2. Microcirculatory perfusion parameters by study arm. This table shows the distribution of patients and mortality rates by study arm included in the ancillary study. Overall, the mortality rates among the groups were similar. (DOCX 13 kb)
\end{abstract}

Additional file 3: Table S3. Microcirculatory perfusion analysis by study arm. In a comparison of study parameters, only MFI was found to have a statistically significant difference between study arms; however, the clinical significance of this small difference is likely minimal. (DOCX $16 \mathrm{~kb}$ )

\section{Abbreviations}

AIDS: Acquired immunodeficiency syndrome; CVP: Central venous pressure; DBP: Diastolic blood pressure; ED: Emergency department; EGDT: Early goaldirected therapy; GEE: Generalized estimating equation; ICU: Intensive care unit; MAP: Mean arterial pressure; MFI: Microcirculatory flow index; PPV: Proportion of perfused vessels; ProCESS: Protocolized Care for Early Septic Shock study; PVD: Perfused vascular density; SBP: Systolic blood pressure; $\mathrm{SCVO}_{2}$ : Central venous oxygen saturation; SOFA: Sequential Organ Failure Assessment; TVD: Total vascular density

\section{Acknowledgements}

We thank the research and clinical staff at the participating institutions for their contributions to this project.

The members of the ProCESS Trial are as follows: Coordinating Center: Derek C. Angus, Amber E. Barnato, Tammy L. Eaton, Elizabeth Gimbel, David T. Huang, Christopher Keener, John A. Kellum, Kyle Landis, Francis Pike, Diana K. Stapleton, Lisa A. Weissfeld, Michael Willochell, Kourtney A. Wofford, Donald M. Yealy. Recruiting Centers: (Site Principal Investigators are listed in Italics): Advocate Christ Medical Center, Oak Lawn, IL: Erik Kulstad, Hannah Watts. Allegheny General Hospital, Pittsburgh, PA: Arvind Venkat. Brigham and Women's Hospital, Boston, MA: Peter C. Hou, Anthony Massaro, Siddharth Parmar. Duke University Medical Center, Durham, NC: Alexander T. Limkakeng, Jr. East Carolina University, Greenville, NC: Kori Brewer, Theodore R. Delbridge, Allison Mainhart. George Washington University Medical Center, Washington, DC: Lakhmir S. Chawla. Hennepin County Medical Center, Minneapolis, MN: James R. Miner. Intermountain Medical Center, Murray, UT: Todd L. Allen, Colin K. Grissom, Los Angeles County + USC Medical Center, Los Angeles, CA: Stuart Swadron. Louisiana State University Health Sciences Center, Shreveport, LA: Steven A. Conrad. Maricopa Medical Center, Phoenix, AZ: Richard Carlson, Frank LoVecchio. Massachusetts General Hospital, Boston, MA: Ednan K. Bajwa, Michael R. Filbin. Blair A. Parry. Methodist Research Institute, Indianapolis, IN: Timothy J. Ellender. North Shore University Hospital, Manhasset, NY: Andrew E. Sama. Norwalk Hospital Norwalk, CT: Jonathan Fine. Penn State Hershey College of Medicine, Hershey, PA: Soheil Nafeei, Thomas Terndrup, Margaret Wojnar. Stanford University School of Medicine, Stanford, CA: Ronald G. Pearl. Summa Health System, Akron, OH: Scott T. Wilber. SUNY Downstate Medical Center, Brooklyn, NY: Richard Sinert. Tampa General Hospital, Tampa, FL: David J. Orban, Jason W. Wilson. Temple University Hospital, Philadelphia, PA: Jacob W. Ufberg. UC Davis Medical Center, Sacramento, CA: Timothy Albertson, Edward A. Panacek. University Medical Center Brackenridge, Austin, TX: Sohan Parekh. UPMC Presbyterian/Shadyside, Pittsburgh, PA: Scott R. Gunn, Jon S. Rittenberger, Richard J. Wadas. University of Alabama at Birmingham, Birmingham, AL: Andrew R. Edwards, Matthew Kelly, Henry E. Wang, University of Arkansas for Medical Sciences, Little Rock, AR: Talmage M. Holmes. University of Maryland at Baltimore, Baltimore, MD: Michael T. McCurdy. University of Minnesota Medical Center, Fairview, MN: Craig Weinert. University of Utah Health Sciences Center, Salt Lake City, UT: Estelle S. Harris. Vanderbilt University Medical Center, Nashville, TN: Wesley H. Self, Diane Dubinski. Washington Hospital Center, Washington, DC: Carolyn A. Phillips, Ronald M. Migues. 


\section{Funding}

This study was sponsored by National Institutes of Health through National Heart, Lung, and Blood Institute grant R01 HL091757 and National Institute of General Medical Sciences grant P50 GM076659.

\section{Availability of data and materials}

The datasets used and/or analyzed during the current study are available from the corresponding author on reasonable request.

\section{Authors' contributions}

MJM and NIS participated in manuscript conception, data acquisition, analysis, and drafting of the manuscript. All of the other authors assisted with data interpretation and drafting of the manuscript. All authors read and approved the final manuscript.

\section{Authors' information}

No additional information provided.

\section{Ethics approval and consent to participate}

The Beth Israel Deaconess Medical Center Committee for Clinical Investigations and each site's review board approved the study design. Each subject or legal representative gave written informed consent.

\section{Consent for publication}

Not applicable.

\section{Competing interests}

No nonfinancial conflicts of interest exist for any of the authors. NIS and MJM have previously received equipment support from MicroVision Medical BV.

\section{Publisher's Note}

Springer Nature remains neutral with regard to jurisdictional claims in published maps and institutional affiliations.

\section{Author details}

'Department of Emergency Medicine and Center for Vascular Biology Research, Beth Israel Deaconess Medical Center, 1 Deaconess Road, CC2-W, Boston, MA 02215, USA. ²Department of Emergency Medicine, Brigham and Women's Hospital, Boston, MA, USA. ${ }^{3}$ Department of Emergency Medicine, Massachusetts General Hospital, Boston, MA, USA. ${ }^{4}$ Department of Emergency Medicine, University of Alabama at Birmingham, Birmingham, AL, USA. ${ }^{5}$ Division of General Medicine, Department of Medicine, Beth Isarel Deaconess Medical Center, Boston, MA, USA. ${ }^{6}$ Department of Critical Care Medicine, University of Pittsburgh School of Medicine, Pittsburgh, PA, USA. ${ }^{7}$ Division of Molecular Medicine, Department of Medicine, and Center for Vascular Biology Research, Beth Israel Deaconess Medical Center, Boston, MA USA. ${ }^{8}$ Clinical Research Center, Soroka University Medical Center, Be'er-Sheva, Israel. ${ }^{9}$ Center for Critical Care Services, Cooper University Hospital, Camden, NJ, USA. ${ }^{10}$ Department of Emergency Medicine, University of Pittsburgh School of Medicine, Pittsburgh, PA, USA.

\section{Received: 5 April 2018 Accepted: 15 October 2018}

Published online: 20 November 2018

\section{References}

1. Ince C. The microcirculation is the motor of sepsis. Crit Care. 2005;9(Suppl 4):S13-9.

2. Lam C, Tyml K, Martin C, Sibbald W. Microvascular perfusion is impaired in a rat model of normotensive sepsis. J Clin Invest. 1994;94:2077-83.

3. De Backer D, Creteur J, Preiser JC, Dubois MJ, Vincent JL. Microvascular Blood Flow Is Altered in Patients with Sepsis. Am J Respir Crit Care Med. 2002;166:98-104

4. Sakr Y, Dubois MJ, De Backer D, Creteur J, Vincent JL. Persistent microcirculatory alterations are associated with organ failure and death in patients with septic shock. Crit Care Med. 2004;32:1825-31.

5. Vincent $J \mathrm{~L}$, De Backer D. Microvascular dysfunction as a cause of organ dysfunction in severe sepsis. Crit Care. 2005;9(Suppl 4):S9-12.

6. Trzeciak S, Rivers EP. Clinical manifestations of disordered microcirculatory perfusion in severe sepsis. Crit Care. 2005;9(Suppl 4):S20-6.
7. Sakr Y, Chierego M, Piagnerelli M, Verdant C, Dubois MJ, Koch M, Creteur J, Gullo A, Vincent JL, De Backer D. Microvascular response to red blood cell transfusion in patients with severe sepsis. Crit Care Med. 2007;35:1639-44.

8. Trzeciak S, Dellinger RP, Parrillo JE, Guglielmi M, Bajaj J, Abate NL, Arnold RC, Colilla S, Zanotti S, Hollenberg SM, Microcirculatory Alterations in Resuscitation and Shock Investigators. Early microcirculatory perfusion derangements in patients with severe sepsis and septic shock: relationship to hemodynamics, oxygen transport, and survival. Ann Emerg Med. 2007;49: 88-98.e2.

9. Trzeciak S, Cinel I, Phillip Dellinger R, Shapiro NI, Arnold RC, Parrillo JE, Hollenberg SM, Microcirculatory Alterations in Resuscitation and Shock (MARS) Investigators. Resuscitating the microcirculation in sepsis: the central role of nitric oxide, emerging concepts for novel therapies, and challenges for clinical trials. Acad Emerg Med. 2008;15:399-413.

10. Trzeciak S, McCoy JV, Phillip Dellinger R, Arnold RC, Rizzuto M, Abate NL, Shapiro NI, Parrillo JE, Hollenberg SM, Microcirculatory Alterations in Resuscitation and Shock (MARS) Investigators. Early increases in microcirculatory perfusion during protocol-directed resuscitation are associated with reduced multi-organ failure at $24 \mathrm{~h}$ in patients with sepsis. Intensive Care Med. 2008;34:2210-7.

11. Arnold RC, Parrillo JE, Phillip Dellinger R, Chansky ME, Shapiro NI, Lundy DJ, Trzeciak S, Hollenberg SM. Point-of-care assessment of microvascular blood flow in critically ill patients. Intensive Care Med. 2009:35:1761-6.

12. Ospina-Tascon G, Neves AP, Occhipinti G, Donadello K, Buchele G, Simion D, Chierego ML, Silva TO, Fonseca A, Vincent JL, De Backer D. Effects of fluids on microvascular perfusion in patients with severe sepsis. Intensive Care Med. 2010;36:949-55.

13. Taccone FS, Su F, Pierrakos $C$, He X, James $S$, Dewitte $O$, Vincent $J$, De Backer D. Cerebral microcirculation is impaired during sepsis: an experimental study. Crit Care. 2010;14:R140.

14. Kanoore Edul VS, Enrico C, Laviolle B, Vazquez AR, Ince C, Dubin A. Quantitative assessment of the microcirculation in healthy volunteers and in patients with septic shock. Crit Care Med. 2012;40:1443-8.

15. Spronk PE, Ince C, Gardien MJ, Mathura KR, Oudemans-van Straaten HM, Zandstra DF. Nitroglycerin in septic shock after intravascular volume resuscitation. Lancet. 2002;360:1395-6.

16. Acheampong A, Vincent $J$. A positive fluid balance is an independent prognostic factor in patients with sepsis. Crit Care. 2015;19:251.

17. De Backer D, Creteur J, Dubois MJ, Sakr Y, Koch M, Verdant C, Vincent JL. The effects of dobutamine on microcirculatory alterations in patients with septic shock are independent of its systemic effects. Crit Care Med. 2006;34: 403-8.

18. Massey MJ, Shapiro NI. A guide to human in vivo microcirculatory flow image analysis. Crit Care. 2016;20:35.

19. Groner W, Winkelman JW, Harris AG, Ince C, Bouma GJ, Messmer K, Nadeau RG. Orthogonal polarization spectral imaging: a new method for study of the microcirculation. Nat Med. 1999:5:1209-12.

20. Goedhart PT, Khalilzada M, Bezemer R, Merza J, Ince C. Sidestream dark field (SDF) imaging: a novel stroboscopic LED ring-based imaging modality for clinical assessment of the microcirculation. Opt Express. 2007;15:15101-14.

21. McCoy J, Trzeciak S, Parrillo J, Dellinger RP, Shapiro N, Abate N, Arnold R, Hollenberg S. Improved organ function at 24 hours is associated with increased microcirculatory flow during the early resuscitation of patients with sepsis [abstract]. Acad Emerg Med. 2007;14:S10-1.

22. De Backer D, Donadello K, Sakr Y, Ospina-Tascon G, Salgado D, Scolletta S, Vincent JL. Microcirculatory alterations in patients with severe sepsis: impact of time of assessment and relationship with outcome. Crit Care Med. 2013; 41:791-9.

23. Top AP, Ince C, de Meij N, van Dijk M, Tibboel D. Persistent low microcirculatory vessel density in nonsurvivors of sepsis in pediatric intensive care. Crit Care Med. 2011;39:8-13.

24. ProCESS Investigators, Yealy DM, Kellum JA, Huang DT, Barnato AE, Weissfeld LA, Pike F, Terndrup T, Wang HE, Hou PC, LoVecchio F, Filbin MR, Shapiro NI, Angus DC. A randomized trial of protocol-based care for early septic shock. N Engl J Med. 2014;370:1683-93.

25. Rivers E, Nguyen B, Havstad S, Ressler J, Muzzin A, Knoblich B, Peterson E, Tomlanovich M, Early Goal-Directed Therapy Collaborative Group. Early goal-directed therapy in the treatment of severe sepsis and septic shock. N Engl J Med. 2001;345:1368-77.

26. Bone RC. American College of Chest Physicians/Society of Critical Care Medicine Consensus Conference: definitions for sepsis and organ failure 
and guidelines for the use of innovative therapies in sepsis. Crit Care Med. 1992;20:864-74.

27. Massey MJ, Larochelle E, Najarro G, Karmacharla A, Arnold R, Trzeciak S, Angus DC, Shapiro NI. The microcirculation image quality score:

development and preliminary evaluation of a proposed approach to grading quality of image acquisition for bedside videomicroscopy. J Crit Care. 2013;28:913-7.

28. Filbin MR, Hou PC, Massey M, Barche A, Kao E, Bracey A, Skibsted S, Chang $Y$, Shapiro NI. The microcirculation is preserved in emergency department low-acuity sepsis patients without hypotension. Acad Emerg Med. 2014;21: 154-62.

29. De Backer D, Hollenberg S, Boerma C, Goedhart P, Buchele G, Ospina-

Tascon G, Dobbe I, Ince C. How to evaluate the microcirculation: report of a round table conference. Crit Care. 2007;11:R101.

30. Bezemer R, Dobbe JG, Bartels SA, Boerma EC, Elbers PW, Heger M, Ince C. Rapid automatic assessment of microvascular density in sidestream dark field images. Med Biol Eng Comput. 2011;49:1269-78.

31. De Backer D, Verdant C, Chierego M, Koch M, Gullo A, Vincent JL. Effects of drotrecogin alfa activated on microcirculatory alterations in patients with severe sepsis. Crit Care Med. 2006;34:1918-24.

Ready to submit your research? Choose BMC and benefit from:

- fast, convenient online submission

- thorough peer review by experienced researchers in your field

- rapid publication on acceptance

- support for research data, including large and complex data types

- gold Open Access which fosters wider collaboration and increased citations

- maximum visibility for your research: over $100 \mathrm{M}$ website views per year

At BMC, research is always in progress.

Learn more biomedcentral.com/submissions 